\title{
Predicted dynamic electrothermal performance of thermistor bolometer radiometers for Earth radiation budget applications
}

\author{
Martial P. A. Haeffelin, J. Robert Mahan, and Kory J. Priestley
}

\begin{abstract}
The Earth Radiation Budget Experiment (ERBE) and the Clouds and the Earth's Radiant Energy System (CERES) rely on scanning thermistor bolometer radiometers of a similar design for accomplishing their mission. High-level dynamic electrothermal models of these instruments have been developed on the basis of the Monte Carlo ray-trace, finite-difference, and finite-element methods. The models are capable of simulating the end-to-end response of the ERBE and the CERES instruments to simulated sequences of Earth scenes. Such models will prove useful in the design of future generations of similar instruments, in defining ground-based and in-flight calibration and data-reduction strategies, in the interpretation of flight data, and in understanding data anomalies that might arise after the instruments have been placed in orbit. Two modules that make up the end-to-end model are presented: the optical-thermal radiative module and the thermistor bolometer dynamic electrothermal module. The optics module is used to determine the point-spread function of the optics, which establishes that the instrument has sharply defined footprints on the Earth. Results obtained with the thermistor bolometer dynamic electrothermal module provide valuable insights into the details of channel operation and establish its high level of equivalence. The combination of the two modules allows the point-spread function of the instrument to be determined and reveals the potential of this tool for scanning realistic Earth scenes. (C) 1997 Optical Society of America
\end{abstract}

\section{Introduction}

The last third of the 20th century has been characterized by an increased awareness of man's impact on the environment. ${ }^{1}$ Initial interest in the role of greenhouse gases ${ }^{2}$ and more recent interest in the role of cloud forcing ${ }^{3}$ have led to ambitious programs to monitor the Earth's global radiative energy budget from platforms orbiting in space. Two of these programs, the Earth Radiation Budget Experiment (ERBE) ${ }^{4,5}$ initiated in the mid-1980's and the Clouds and the Earth's Radiant Energy System (CERES) 6,7 scheduled for operation in the late 1990's and beyond, rely on scanning thermistor bolometer radiometers to accomplish their mission.

M. P. A. Haeffelin and J. R. Mahan are with the Virginia Polytechnic Institute and State University, Blacksburg, Virginia 24061-0238. K. J. Priestley is with the NASA Langley Research Center, MS 472, Hampton, Virginia 23681-0001. M. P. A. Haeffelin's current address is NASA Langley Research Center, MS 420, Hampton, Virginia 23681-0001.

Received 15 January 1997; revised manuscript received 13 May 1997.

0003-6935/97/287129-14\$10.00/0

(C) 1997 Optical Society of America
Variations in the Earth's radiative energy budget capable of signaling climate changes are expected to be subtle and to occur over periods of years or even decades. Instruments intended to sense these variations must therefore be capable of providing longterm, continuous data records relatively free of instrument-imposed biases. This requirement has led to an understandable reluctance to change the basic instrument design from one monitoring program to the next. Therefore the ERBE and CERES scanning radiometric channels are quite similar in their design and operation, and it is likely that this generic type of instrument will remain important well into the 21 st century.

\section{ERBE and CERES Scanning Radiometers}

ERBE was started by NASA in the early 1980's to monitor the radiative field of the Earth. Each satellite in the mission carried two types of instruments: narrow fieldof-view scanning thermistor bolometer radiometers and wide field-of-view nonscanning active-cavity radiometers. The description of the nonscanning instruments can be found in Refs. 8 and 9. Although the activecavity instrument is an absolute radiometer of great accuracy, it will not be used in the next-generation mission, 


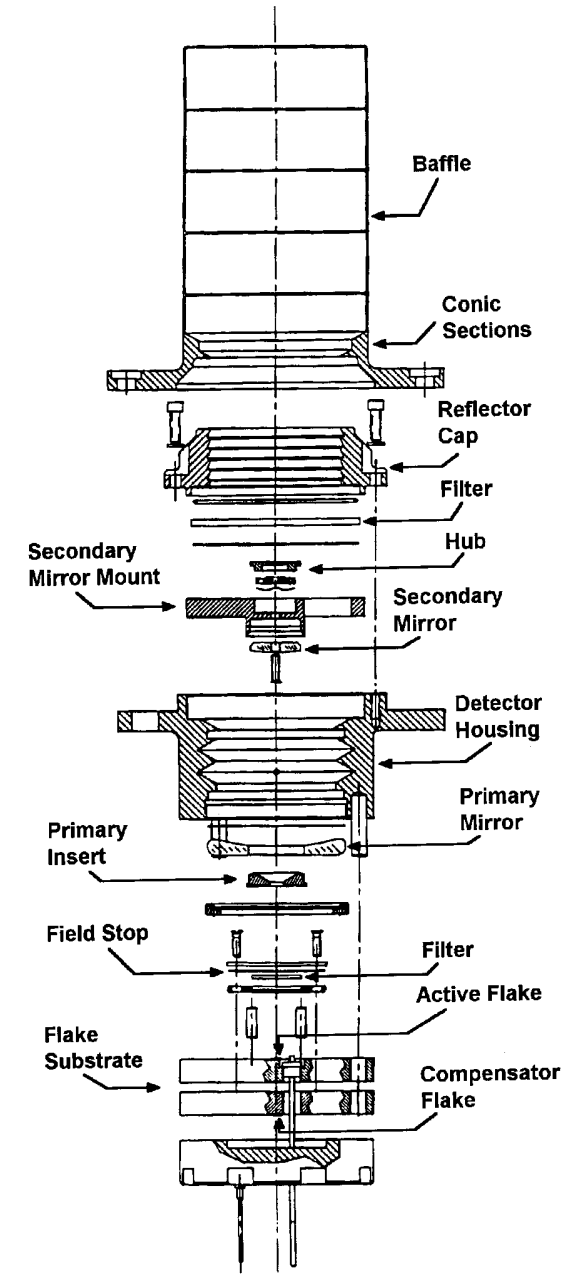

Fig. 1. View of the CERES scanning thermistor bolometer radiometer geometry, courtesy of NASA Langley Research Center.

the CERES. The narrow field-of-view scanning radiometers were found to be more suitable for Earth radiation budget monitoring because of their greater spatial resolution. For studying the effects of clouds on the Earth radiation budget, the pixel size must be sufficiently small to obtain data that are not contaminated by the presence of clouds.

\section{A. Optics}

A scanning thermistor bolometer radiometer typical of those used in ERBE and CERES is depicted in Fig. 1. It consists of a baffled Cassegrain-like telescope that gathers radiation from a restricted Earth scene and focuses it on a thick-film thermistor bolometer. Radiation incident upon the aperture nearly parallel to the instrument optical axis is specularly reflected from the primary and the secondary mirrors before it enters the field stop located behind the primary mirror. The sensing element is mounted behind the field stop on a massive aluminum substrate that serves as a heat sink. Although ERBE radiometers made use of aspherical mirrors, a primary concave paraboloid, and a secondary convex hyperboloid - the true Cassegrain configuration-the mirrors of the
CERES telescope are both spherical and so will be referred to as a Cassegrain-like geometry.

A true Cassegrain geometry allows the spherical aberrations to be eliminated. Thus, if the detector is located correctly, the focus of the image can be good. However, such a geometry increases the off-axis aberrations. The choice of spherical mirrors for CERES was motivated by the necessity to reduce off-axis aberrations. However, this configuration does increase spherical aberrations for radiation parallel to the main axis of the instrument. Because the instrument is not an imager, this has virtually no impact on the quality of the radiometric products.

Bongiovi ${ }^{10}$ showed in his thesis that the optical point-spread function (PSF)-defined in Section 4.A.3- of the CERES radiometer is shaped by the field stop, while the field of view of the ERBE instrument seems to be defined by the telescope optics. This, in part, results from the choice of mirrors. Again the spherical mirrors produce an image with a more uniform aberration throughout all angles of incidence, a situation that creates a more sharply defined PSF. More details on this topic can be found in Ref. 11.

Filters are provided to give the desired spectral sensitivity. Three wavelength intervals are monitored in both ERBE and CERES. The three ERBE scanning channels had an approximately $60-\mathrm{km}$ field of view at nadir and were sensitive in the short $(0.2-$ $5.0 \mu \mathrm{m})$, long $(5.0-50 \mu \mathrm{m})$, and total wavelength bands. The three CERES channels will have approximately twice the spatial resolution of ERBE, and the longwave channel will be replaced by an atmospheric window $(8.0-12.0 \mu \mathrm{m})$ channel.

Although the designs of ERBE and CERES appear similar, the CERES scanning radiometer is a completely new instrument. The geometry of the telescope has been fine-tuned to reduce contamination from off-axis radiation. Bongiovi ${ }^{10}$ compares the optical PSF of the ERBE and CERES telescopes and shows that the CERES telescope is far superior to the ERBE in the definition of the pixel.

\section{B. Detector}

The heart of the scanning radiometric channels in both ERBE and CERES consists of two nearly identical thick-film thermistor bolometer detectors mounted on adjacent faces of a thick aluminum substrate that acts as a heat sink. Temperature changes in the heat sink induced by changes in the instrument thermal environment can be compensated for effectively when the two detectors are placed in adjacent arms of a two-active-arm deflection bridge. The active and compensating detectors are 40- to 50- $\mu \mathrm{m}$-thick wafers, consisting of an absorber layer, a thermistor layer, and a thermalimpedance layer, as shown in Fig. 2. Radiation from the Earth scene is directed onto the active thermistor bolometer detector by the Cassegrain telescope optics.

Radiation incident upon the absorber layer of the active detector is converted into sensible heat and 


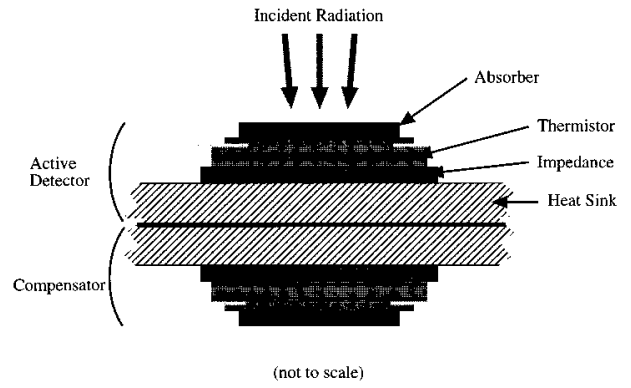

Fig. 2. Cross section of the CERES thermistor bolometer active and compensating detectors.

conducted through the thermistor and thermal impedance to the heat sink, where the temperature is maintained within fixed narrow bounds by a servocontroller. The change in temperature in the thermistor is highly dependent on the rate at which the heat is conducted away to the heat sink. The thermal impedance layer between the thermistor and the heat sink maintains a large temperature gradient that amplifies the temperature changes in the thermistor. The resulting temperature rise in the thermistor produces a corresponding resistance change that is sensed by the deflection bridge circuit. The amplified bridge voltage may then be calibrated in terms of the incident radiation. A schematic diagram of the bridge amplifier circuit appears in Fig. 3.

Thermistor bolometer radiometers scan the Earth from limb to limb with a scan period of the order of a few seconds, and so the incident-radiation signal is highly transient. This means that the thermal and electrical frequency response characteristics intervene in determining the instrument transfer function. In practice, however, radiance data are generally interpreted as if they were instantaneous representations of the scene being viewed. Also, the scenes viewed by these instruments are not necessarily spatially homogeneous; the spatial distribution of the energy incident to the radiation detector will generally vary from scene to scene. However, radiance data are interpreted as if all possible spatial distributions of a given amount of energy from an Earth scene produce the same instrument deflection, i.e., equivalence is assumed. Finally, thermal noise that is emitted and reflected to the detector from the instrument structure varies from orbit to orbit and, to a lesser extent, with the scene being viewed. Most of the thermal noise sources are adequately sampled at the ends of each scan cycle when the instrument observes cold space. This will occur every $3.3 \mathrm{~s}$ in CERES. Scene-dependent variations in thermal noise that may occur during the scan cycle are not sampled.

High-level dynamic electrothermal models of scanning thermistor bolometer radiometers are being developed in support of ERBE and CERES. 9,10,12,13 This effort is motivated by several factors: First, it is interesting to know the impact of potential sources of the measurement error described above, i.e., dynamic response, nonequivalence, and thermal contamination, on the instrument's accuracy and precision. Sufficiently high-level numerical representations of these instruments are fully capable of addressing these issues. Dynamic electrothermal models have also proved useful in the initial design stage for optimizing physical dimensions and surface optical treatments, for sizing and selecting electronic components, and for predicting the resulting instrument PSF. Groundbased and in-flight calibration systems and procedures as well as data-reduction strategies can be designed and tested before actual hardware is available. Finally, these models have promise for use in the interpretation of flight data ${ }^{14}$ and for understanding and dealing with anomalies that may arise after the instrument has been placed in orbit.

\section{High-Level Numerical Models of a CERES-like Scanning Thermistor Bolometer Radiometer}

Our ultimate goal is to produce a high-level end-toend dynamic electrothermal model of scanning thermistor bolometer radiometers of the type used in ERBE and CERES. Such a model would consist of three major blocks: an optical-thermal radiative module, a detector dynamic electrothermal response module, and a structural heat conduction module. The approach has been to develop these three modules separately and then to integrate them into a coherent end-to-end instrument model. We empha-

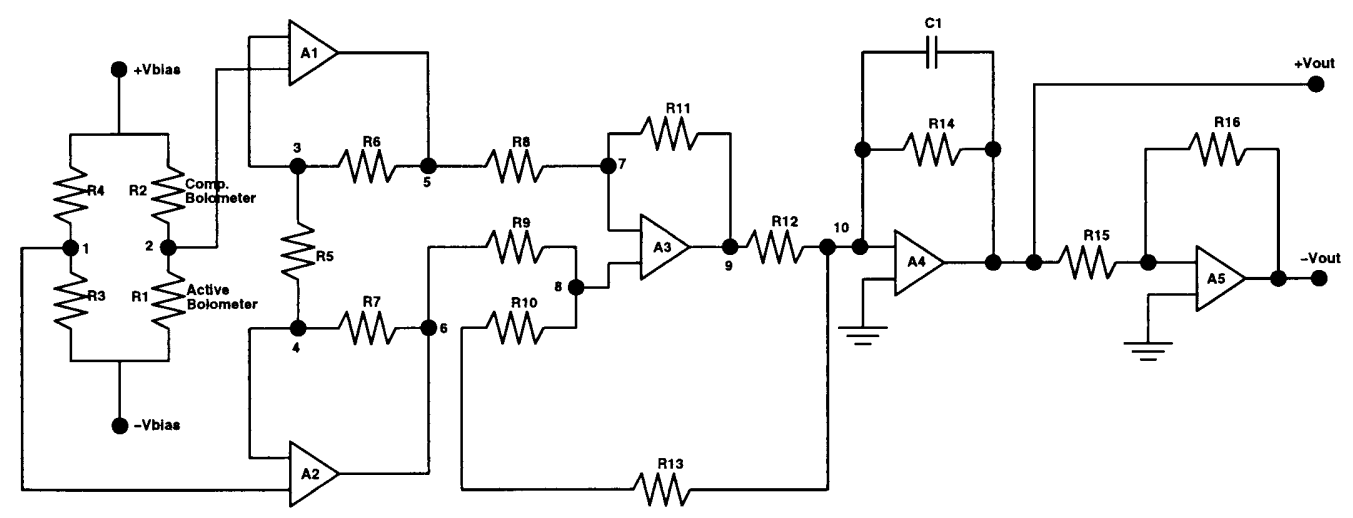

Fig. 3. Bridge amplifier circuit for the CERES scanning radiometer protoflight model. 
size the optical-thermal radiative module and the detector dynamic electrothermal module.

\section{A. Optical-Thermal Radiative Module}

The optical-thermal radiative module is based on the Monte Carlo ray-trace method. Therefore it is limited to wavelengths where diffraction effects are negligible. The dimensions of the ERBE and the CERES instruments are such that diffraction may be deemed negligible for all but the longest wavelengths of interest, i.e., beyond approximately $50 \mu \mathrm{m}$. The error in the overall radiative analysis associated with neglecting diffraction effects is probably less than that associated with uncertainties in the surface radiative properties used in the analysis. This optical module was developed first by Meekins ${ }^{12}$ for the ERBE instrument. Meekins performed a radiative and optical analysis of the ERBE radiometer that gave good insight into the characteristics of the instrument. The model was later modified by Bongiovi ${ }^{10}$ to include the CERES geometry. The geometry taken into account in the model was more complete; the baffle, for instance, was added to the model. Bongiovi computed the radiative and optical characteristics of both the ERBE and the CERES instruments with greater confidence than before, thanks to the advance of computers, which permitted higher speed and accuracy. Finally the model was modified by Haeffelin to provide input to his dynamic electrothermal model of the detector.

Applications of the Monte Carlo ray-trace method in optics and thermal radiation are widely documented in the literature ${ }^{15-21}$; therefore details about the general method are not included here. Briefly, a large number of energy bundles, or rays, are allowed to enter the radiometer through the baffle opening with a desired spatial and spectral distribution. Each ray is traced until it strikes a surface, at which point its fate is determined by the comparison of the local values of the surface optical properties (absorptivity and degree of specularity of the reflectivity) with random numbers whose values are uniformly distributed between zero and unity. For example, if the first random number drawn is less than the local absorptivity, the ray is absorbed; otherwise it is reflected. In this way the surface optical properties are interpreted as probabilities, either that a given ray will be absorbed or reflected or, if reflected, that the reflection will be specular or diffuse. If a reflection turns out to be diffuse, the values of a pair of random numbers are used to determine the direction of the diffuse reflection. Rays emitted from surfaces of the radiometer are treated in a manner similar to rays entering through the baffle opening.

Typically, a ray-trace analysis must be performed for every wavelength band of interest, assuming the optical properties of the enclosure to be constant within each wavelength interval. For the purpose of our study we assume constant optical properties over the entire spectral domain of interest $(0.2-50.0 \mu \mathrm{m})$ to limit computation time. The gray analysis, which is an option in the code, is perfectly adapted to our study.
The interior surfaces of the radiometer are subdivided into surface elements whose size and number depend on the spatial resolution required to achieve accuracy goals. When all the entering and emitted rays either have been traced to a surface element where they are absorbed or have exited the radiometer through the baffle opening, it is possible to compute a quantity called the radiation distribution factor $D_{\mathrm{ij}}$, defined as the fraction of radiation emitted from surface element $i$ (or entering through the opening) that is absorbed by surface element $j$ (or exiting through the opening). After a statistically significant number of rays have been traced, usually of the order of millions depending on the accuracy desired, the distribution factor can be estimated as

$$
D_{\mathrm{ij}} \simeq \frac{N_{\mathrm{ij}}}{N_{\mathrm{i}}},
$$

where $N_{\mathrm{ij}}$ is the number of rays emitted diffusely from surface element $i$ that are absorbed by surface element $\mathrm{j}$, and $N_{\mathrm{i}}$ is the total number of rays emitted from surface element $i$.

The distribution factors $D_{\mathrm{ij}}$ can be shown to have the following properties:

$$
\begin{aligned}
\sum_{\mathrm{j}=1}^{n} D_{\mathrm{ij}} & =1.0, \quad \mathrm{i}=1,2, \ldots, n, \\
\epsilon_{\mathrm{i}} A_{\mathrm{i}} D_{\mathrm{ij}} & =\epsilon_{\mathrm{j}} A_{\mathrm{j}} D_{\mathrm{ji}}, \\
\sum_{\mathrm{i}=1}^{n} \epsilon_{\mathrm{i}} A_{\mathrm{i}} D_{\mathrm{ij}} & =\epsilon_{\mathrm{j}} A_{\mathrm{j}}, \quad \mathrm{j}=1,2, \ldots, n,
\end{aligned}
$$

where $\epsilon$ and $A$ represent emissivity and surface area, respectively. Equation (2) is a statement of conservation of energy and Eq. (3) expresses reciprocity. We obtain Eq. (4) by summing Eq. (3) over i and applying Eq. (2) to the result.

After a statistically valid estimate of the distribution factor has been obtained, it may be used to compute the radiation absorbed by any surface $\mathrm{j}$,

$$
Q_{\mathrm{abs}, \mathrm{j}}=\sum_{\mathrm{i}=1}^{n} A_{\mathrm{i}} \epsilon_{\mathrm{i}} \sigma T_{\mathrm{i}}^{4} D_{\mathrm{ij}}(W) .
$$

In this circumstance a given Earth scene can be interpreted as an appropriate distribution of black point sources with various temperatures located at infinity in front of the baffle opening. After the temperatures of all of the interior surface elements and the point sources representing the Earth scene are specified, Eq. (5) can be used to compute the distribution of absorbed radiation on all internal surfaces of the radiometer, including the active flake.

\section{B. Detector Dynamic Electrothermal Module}

The thermal part of the model characterizes the temperature changes in both the active detector and the compensator flakes, as well as in the substrates, associated with temporal and spatial variations of the radiative input. The electrical part of the model is used to predict the variation of overall resistance of both 
flakes resulting from thermal changes and to compute the signal response of the electronic circuit. The electrical analysis also is used to predict the Joulean heating in both thermistors and pass it to the thermal model. The two parts of the model are fully coupled. The model was developed by Haeffelin and Priestley.

The unsteady three-dimensional temperature field $T(x, y, z, t)$ in the detector flake is given by the solution to the unsteady heat conduction equation,

$$
\frac{\partial}{\partial x}\left(k_{x} \frac{\partial T}{\partial x}\right)+\frac{\partial}{\partial y}\left(k_{y} \frac{\partial T}{\partial y}\right)+\frac{\partial}{\partial z}\left(k_{z} \frac{\partial T}{\partial z}\right)+\dot{q}=\rho C \frac{\partial T}{\partial t},
$$

subject to appropriate initial and boundary conditions. In Eq. (6), $k_{x}, k_{y}$, and $k_{z}$ are the thermal conductivities in the $x, y$, and $z$ directions; $\dot{q}$ is a volumetric heat source term; $\rho$ is the mass density; and $C$ is the specific heat.

Two-dimensional electrical diffusion in the thermistor layer is described by the Laplace equation,

$$
\frac{\partial}{\partial x}\left(\sigma_{e} \frac{\partial V}{\partial x}\right)+\frac{\partial}{\partial y}\left(\sigma_{e} \frac{\partial V}{\partial y}\right)=0,
$$

derived from the continuity equation for the current density field $\mathbf{J}$,

$$
\boldsymbol{\nabla}(\mathbf{J})=0 .
$$

In Eq. (7), $V$ is the electric potential and $\sigma_{e}$ is the electrical conductivity. A two-dimensional model is used for electrical diffusion because the thermistor layer is electrically insulated in the vertical direction by two layers of epoxy.

Both thermal and electrical diffusion in the detector flake are represented by a fully implicit finitedifference formulation. The finite-difference method is conceptually simple and straightforward, and it is well suited to the rectangular geometry of the detector flake. Because the electrical and thermophysical properties of the various layers are different, the finite-difference analysis is developed initially from the point of view of control volumes. Each layer is first subdivided into control volumes such that the properties are uniform within a given control volume. The nodes are located at the centers of the control volumes, and each node has six neighbors. The finite-difference nodal mesh for the CERES-like detector flake of Fig. 2 is shown in Fig. 4 . The number of layers of nodes through the flake is fixed, but the number of nodes per layer is variable.

In an unsteady finite-difference formulation, the temperature of a given node at time $t$ is directly related to the temperatures of its six neighbors and to its own temperature at the previous time $(t-\delta t)$ by an algebraic approximation to Eq. (6). The degree of approximation depends on the finite-difference grid spacing and the size of the time steps used as well as the convergence criteria used for each time step. The details of this process are widely available in standard texts, of which Ref. 22 is an example. The details of the current application are given in Ref. 13 .
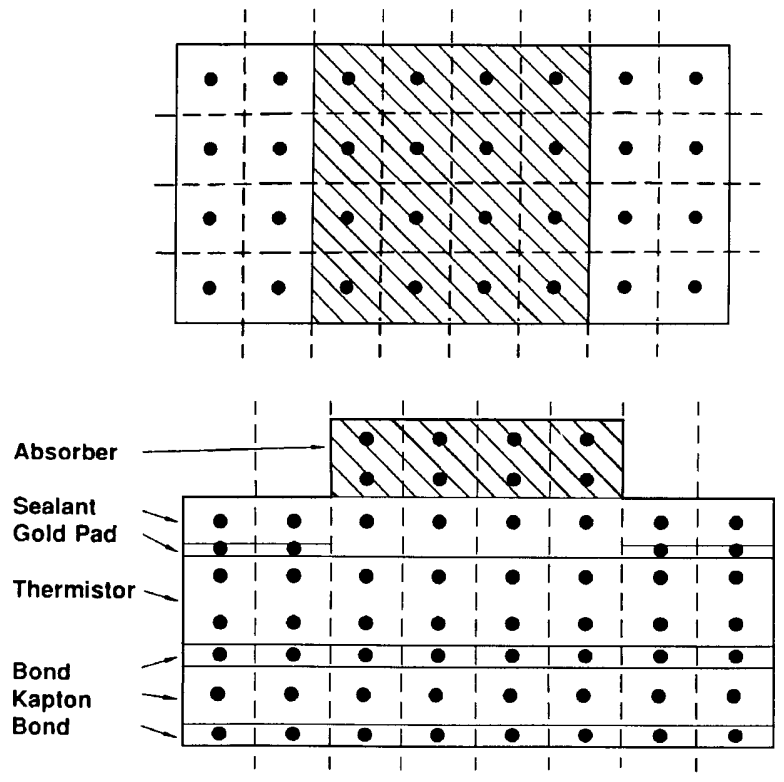

Fig. 4. Plan (top) and elevation (bottom) views of the finitedifference nodal mesh for transient thermal and electrical analysis of the CERES bolometer detector.

The detector flake represented in Figs. 2 and 4 is geometrically three-dimensional. Although we know that heat is conducted principally in one direction, the three-dimensionality of the problem is retained to maintain the flexibility of the model and to sample the effects of three-dimensionality. Equation (6) is integrated over time and over each control volume to obtain a discretized representation of the heat diffusion equation. The resulting algebraic difference equations of the problem are solved by a GaussSiedel point-by-point method. The solution starts with an initial temperature, usually $311 \mathrm{~K}$, for the entire grid shown in Fig. 4. An initial temperature guess is assigned to each node to calculate the node temperatures at the first time step. Each grid point, or node, is visited successively to calculate the temperature at that point with the discretized heat diffusion equation. In this step the temperatures of the six neighbors are needed and so the most recent values are used. When the entire grid has been visited in this manner, one iteration has been completed. Iterations are performed successively until the change in node temperatures from one iteration to the next becomes less than an acceptable tolerance.

The active and compensator detector flakes, both of which are analyzed in this manner, are connected in the detector bridge circuit as shown in Fig. 3. In each flake the current passes through the thermistor layer connected to the circuit by platinum leads. The resistance provided by a square sheet of thermistor is related to its temperature by

$$
R=R_{0} \exp \left[B\left(\frac{1}{T}-\frac{1}{T_{0}}\right)\right],
$$

where $R_{0}$ is the known thermistor resistance at temperature $T_{0}, \alpha$ is the temperature coefficient of resis- 
tance around $T_{0}$, and $B$ is the activation energy of the thermistor material. For a rectangular crosssection conductor of thickness $\delta$, length $L$, and width $w$, the electrical conductivity $\sigma_{e}$ is related to the electrical resistance $R$ by

$$
\sigma_{e}=\frac{L}{R w \delta} .
$$

Equations (9) and (10) are applied to a $\delta \times L \times w$ rectangular volume element to compute the local electrical conductivity from local temperatures provided by the thermal analysis.

When the active flake receives a radiative heat input, its temperature distribution changes rapidly, which in turn changes the overall resistance of the thermistor. Equation (9) is nonlinear in temperature and so cannot be used to relate the average temperature of the thermistor to its overall resistance. Instead, for the value of electrical potential difference across the flake at the beginning of a given calculation time step, a discrete electric field in the thermistor layer is computed with the twodimensional electrical diffusion equation, Eq. (7), and the local values of the electrical conductivity are obtained with Eqs. (9) and (10). We then compute the two-dimensional current density field passing through the flake by applying Ohm's law locally. Finally, the overall electrical resistance of the thermistor is computed as the ratio of the applied potential difference to the computed total current flowing out of (or into) the flake.

When the resistances of the active and compensating detector flakes are known, the signal response of the detector bridge amplifier circuit can be computed. At a given time step with given values for each component of the circuit, resistors, capacitors, and operational amplifiers, the electrical potential $V_{\text {out }}$ in Fig. 3 can be computed. Its dynamic behavior is ruled by a differential equation,

$$
\frac{\mathrm{d} V_{\text {out }}}{\mathrm{d} t}+C_{1} V_{\text {out }}=C_{2}
$$

where $C_{1}$ and $C_{2}$ are constants whose values depend on the values of the circuit components shown in Fig. 3. Equation (11), which is based on successive applications of Kirchhoff's current law to all the nodes in Fig. 3, is solved. The operational amplifiers are assumed to be ideal amplifiers, which implies that the electrical potential at the output of the amplifier is computed as directly proportional to the difference between the electrical potential at the inverting input and that at the noninverting input,

$$
V_{\text {out }}=A\left(V_{\text {in }}{ }^{+}-V_{\text {in }}{ }^{-}\right),
$$

where $A$ is the gain of the amplifier. The signal response of the detector circuit, $V_{\text {out }}$, is then computed as

$$
V_{\text {out }}=C_{3} \exp \left(-C_{1} t\right)+\frac{C_{2}}{C_{1}}
$$

We obtained the signal response of the circuit at each time step by successively invoking the combined thermal and electrical analyses for the radiative input condition prevailing at the beginning of the time step. With sufficiently small time steps, of the order of $1 \mathrm{~ms}$, the continuous response of the thermistor bolometer radiometer to a sequence of simulated Earth scenes can be obtained.

The end-to-end model was also meant to include a module that characterized the heat diffusion in the instrument structure. However, a commercial software package was chosen to perform this task, thereby precluding its integration with the other two modules. The impact of change in thermal noise across a scan has been shown to contribute negligibly to the signal response of the instrument. ${ }^{23}$ Therefore integration of the thermal conductive module into the end-to-end model is not critical and is not discussed further in this research. The end-to-end dynamic radiative and electrothermal model of the entire radiometric channel is thus considered to be complete because the optical-thermal radiative module and the thermistor bolometer dynamic electrothermal module are fully integrated, and the telescope structural dynamic thermal module can be used offline to verify that its integration is unnecessary.

\section{Predicted Channel Performance with the Model}

The optical-thermal radiative module and the thermistor bolometer dynamic electrothermal module have been used separately and in combination to study the optical performance of a radiometer, including thermal radiative contamination and the equivalence and dynamic response characteristics of a thermistor bolometer. Studies with the structure dynamic thermal model have established that the results presented here are not influenced by heat diffusion in the radiometer structure.

\section{A. Predicted Optical-Thermal Radiative Performance}

Three streams of radiation arrive at the active detector during channel operation: (1) direct radiation from the Earth scene that has followed the theoretical optical path defined by the baffle, telescope optics, filters, and a precision field stop (mounted just above the detector in Fig. 2); (2) indirect radiation from the hemispherical space above the baffle opening, some of which is from the intended Earth scene but does not follow the theoretical optical path and the remainder of which is out-of-field radiation; and (3) radiation emitted from the radiometer structure, which is potential thermal radiative contamination. The Monte Carlo ray-trace method allows the radiation that arrives at the active detector to be classified into these three categories.

The fidelity of the modeling tool used to predict the performance has been established by Bongiovi. ${ }^{10}$ The fidelity of a Monte Carlo ray-trace analysis is measured by its degree of convergence and by the statistics of its internal consistency. Degree of convergence refers to the number of energy bundles, or rays, that must be traced in order that the result 
obtained is no longer sensitive to the number of rays traced; and statistics of internal consistency refers to the statistical measure of the degree to which the analysis is consistent with the fundamental laws of radiative transfer such as conservation of energy and reciprocity.

For the analysis of results that are reported here, the CERES-like radiometer is divided into 746 surface elements. Nominal values of the surface optical properties (absorptivity and degree of specularity of the reflectivity) have been used for the various surface coatings and treatments (Chemglaze by Hughson Chemicals, copper black, electrodeposited nickel, polished aluminum, and Black Velvet by 3M). See Ref. 10 for the surface optical properties.

\section{Discretization of the Field of View}

Recall from Section 3.A that a study by Bongiovi ${ }^{10}$ showed that the field of view of the CERES scanning radiometer was defined predominantly by the shape and the dimensions of the field stop. Two approaches are available to characterize the field of view of the instrument with the optics module of the end-to-end model. To define the field of view and the optical PSF (see Section 4.A.3), Bongiovi ${ }^{10}$ used a reverse Monte Carlo ray-trace method, in which energy bundles are diffusely emitted from the detector and those leaving the instrument through the aperture are accounted for. This method does not allow the field of view to be discretized and, hence, heterogeneous scenes cannot be studied. In addition, in Bongiovi's study, the detector was not discretized; hence results obtained from the optical model could not be used as input to the electrothermal model.

Because the field of view is shaped by the field stop, it was decided to base its angular discretization according to the two angles that characterize the field stop: the scan and the cross-scan angles. Note that in the case of a cross-track scanning radiometer, the cross-scan direction corresponds to the alongtrack direction. The scan and cross-scan angles are measured from the instrument optical axis in the scan and cross-scan directions, respectively. In the physical dimensions of the field stop, the maximum scan angle is $\pm 0.65^{\circ}$ and the maximum cross-scan angle is $\pm 1.30^{\circ}$. The discretization is obtained with a $0.0635^{\circ}$ increment and $0.1^{\circ}$ increment, respectively. The increment in the scan direction is defined according to the scan rate of the CERES instrument, which is approximately $63.5 \mathrm{deg} / \mathrm{s}$, and the numerical time step of the electrothermal module, $0.001 \mathrm{~s}$. Therefore, a $0.0635^{\circ}$ increment allows the field of view of the instrument to be updated at every time step when the end-to-end model is used to simulate a scan across a given scene. The increment in the crossscan direction is chosen so that the number of angular intervals is similar to the number of intervals in the scan direction. The angles are not limited to the values that correspond to the physical dimensions of the field stop because some out-of-field radiation reaches the detector through nonoptical paths.

The unit vector that characterizes the direction of

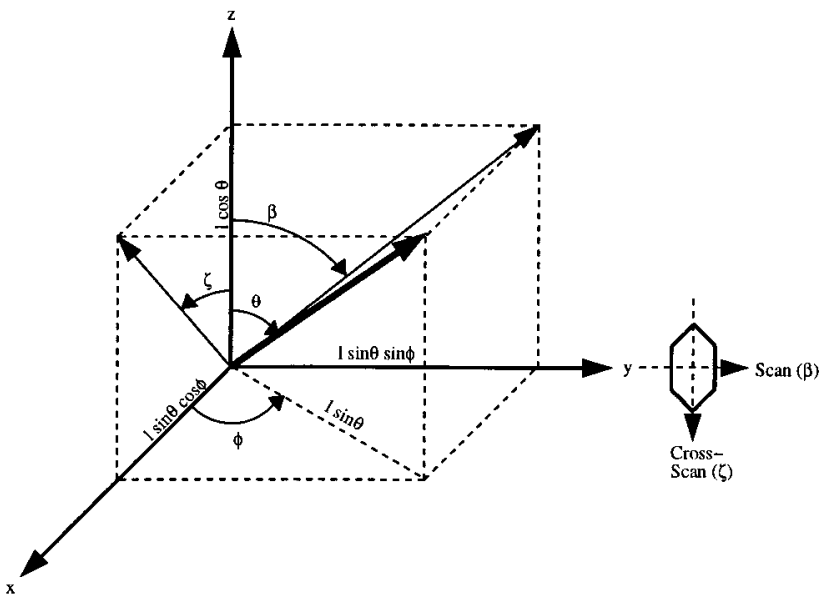

Fig. 5. Geometry for conversion from cross-scan (along-track) and scan (cross-track) angles to zenith and azimuth angles.

collimated radiation incident upon the aperture can be defined by two angles in the spherical coordinate system of the instrument, $\theta$ and $\phi$, respectively, called the zenith and azimuth angles. An angular bin in the field of view of the instrument is defined by a scan angle and a cross-scan angle, respectively $\beta$ and $\zeta$, to which correspond two angles $\theta$ and $\phi$ that define the direction of radiation for that bin. Because the angular bin is small, radiation associated with one bin is assumed to be collimated. According to Fig. 5, the azimuth angle can be defined as

$$
\phi=\arctan \left(\frac{\tan \beta}{\tan \zeta}\right),
$$

and the zenith angle as

$$
\theta=\arctan \left(\frac{\tan \beta}{\sin \phi}\right) \text {. }
$$

The angle $\theta$ is defined between 0 and $\pi$ and the angle $\phi$ is between 0 and $2 \pi$.

The maximum zenith angle to be considered for this application is less than $2^{\circ}$; hence $\cos \theta$ is essentially equal to unity. Therefore the solid angle, defined as "the area intercepted on a unit sphere by a conical angle originating at the sphere center (nondimensionalized by the radius square of the sphere)" 24 can be considered as an area intercepted on the plane tangent to the sphere at the intercept with the cone axis. Hence the solid angle associated with each angular bin, $\Delta \Omega$, is to an acceptable approximation the product of the scan-angle increment and the cross-scan-angle increment,

$$
\Delta \Omega=\Delta \beta \Delta \zeta .
$$

\section{Computation of Distribution Factors}

Distribution factors, defined in Section 3.A, are used to determine the radiative exchange between elements that make up an enclosure. Distribution factors vary according to the conditions under which 
they are developed. Some distribution factors have been computed in enclosures that have spectral characteristics and can be applied only in specific wavelength intervals. Other distribution factors correspond to the energy exchange between two surfaces, but for only limited angular conditions.

To define the field of view of the scanning radiometer, the optics module is used to trace radiation incident upon the instrument aperture through the telescope to the detector. To be able to study heterogeneous Earth scenes, radiation associated with each angular bin is traced independently. For each combination of scan and cross-scan angles, the angles $\theta$ and $\phi$ are computed with Eqs. (14) and (15), and collimated rays are emitted in direction $\theta, \phi$ from the imaginary surface of the instrument aperture and traced through the telescope. Because a fairly large fraction of the radiation incident upon the instrument aperture actually reaches the detector, approximately $30 \%$ for on-axis radiation, only 40,000 bundles need to be traced to obtain a good definition of the spatial distribution of energy on the detector.

On the basis of Eq. (1), distribution factors are computed for each angular bin as

$$
D_{\text {elt }}^{\text {bin }} \simeq \frac{N_{\text {elt }}^{\text {bin }}}{N^{\text {bin }}},
$$

where $N_{\text {elt }}^{\text {bin }}$ is the number of rays emitted in the collimated direction of a given bin from the instrument aperture absorbed by a given surface element on the detector, and $N^{\text {bin }}$ is the total number of rays emitted from the instrument aperture in the collimated direction of the bin.

Figure 6(a) shows the spatial distribution of energy absorbed in the plane of the detector for collimated radiation that enters the instrument aperture parallel to the optical axis. The three legs supporting the secondary mirror are clearly visible. The image is nearly circular, which corresponds to the geometry of the baffle opening. The image is centered on the detector and has a 0.1-mm diameter. For collimated on-axis radiation, the field-stop aperture does not intercept any radiation.

The grid shown in Fig. 6(a) is that of the elements that define the mesh of the electrothermal model. As described in Section 3.B, the surface of the absorber layer of the detector has been discretized in a $16 \times 16$ grid, or 256 elements, of which only the central 16 are visible in Fig. 6(a). Figure 6(b) shows the distribution factors that correspond to the spatial distribution of Fig. 6(a). Note that, although the size of the image of Fig. 6(a) is approximately that of one element, four elements are actually illuminated because the image is centered. The remaining elements receive virtually no radiation.

For each angular bin, 256 distribution factors are computed and stored. As the scan and cross-scan angles are incremented, the limit of the field of view is found when all the distribution factor values are zero. Note that the spatial discretization of the detector in 256 elements is necessary only when the

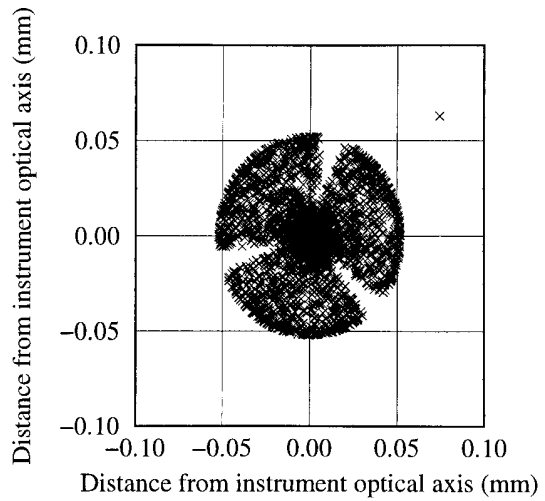

(a)

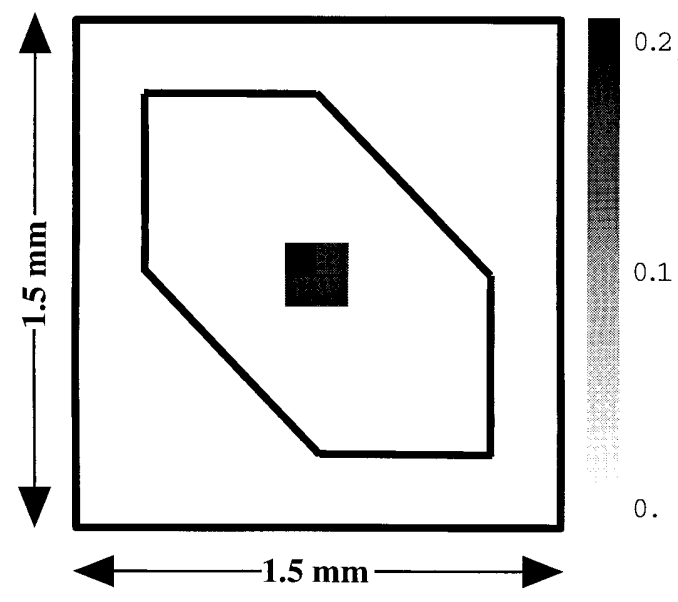

(b)

Fig. 6. Spatial distribution of energy absorbed in the plane of the detector for collimated radiation parallel to the optical axis of the telescope: (a) energy bundle distribution, (b) distribution factors.

optics module serves as an input to the detector electrothermal model. For strictly optical and radiative considerations, the absorbing surface of the active detector can be considered as one element. In this case the distribution factor that represents the fraction of energy incident upon the instrument aperture within a given angular bin that reaches the active detector can be computed as

$$
D^{\text {bin }}=\sum_{\text {elt }=1}^{256} D_{\text {elt }}^{\text {bin }} .
$$

In Eq. (18), $D_{\text {elt }}^{\text {bin }}$ is the distribution factor for each of the 256 elements of the detector. The sum is therefore carried out on the 256 elements.

\section{Point-Spread Function of the Optics}

The distribution factors derived for collimated radiation define the spatial distribution of a distant object in the plane containing the detector. In other words, given a collimated flux originating from a distant point source, the collimated distribution factors define the spatial distribution of the image in the plane of the detector. This is the definition of a PSF. The 

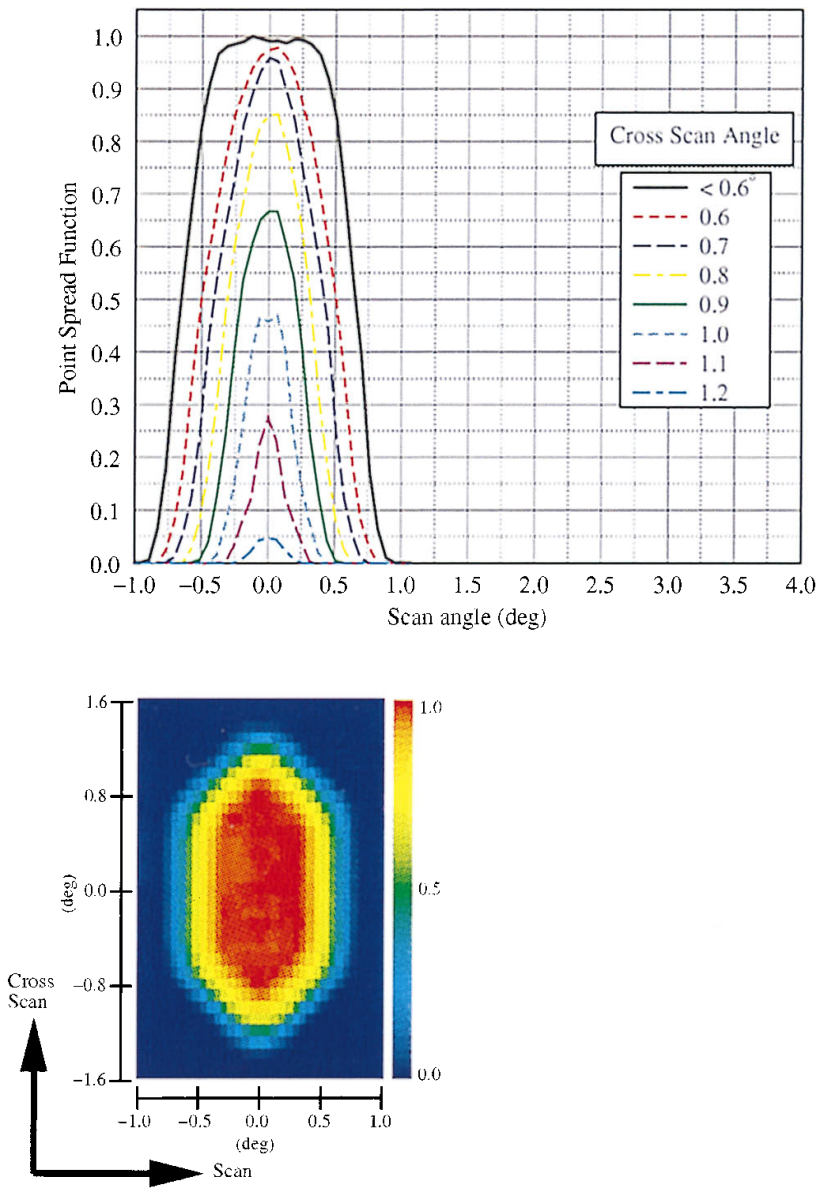

Fig. 7. PSF of the optics: (top) one-dimensional representation, (bottom) two-dimensional representation.

PSF of the optics-i.e., staring instrument-can also be used in combination with the image flux for retrieving the distribution of the object flux. More details can be found in Ref. 9 .

The collimated distribution factors of Eq. (18) for all considered angular bins define the PSF of the optics because the PSF shows the relative contribution of a uniform array of point sources to the total radiation incident upon the plane of the detector. Figure 7 shows the PSF of the optics. We normalized the PSF by dividing each contribution by the maximum value of the distribution. Figure 7(a) gives a one-dimensional view of the PSF in the scan direction, where each curve is associated with a specific cross-scan angle. Figure 7(b) represents a two-dimensional distribution with the relative contribution of each point source defined by an angular bin. This figure shows that the field of view of the instrument is very sharply defined. The value of the PSF is greater than 0.9 over approximately $40 \%$ of the field of view-red area of Fig. 7(b). The field of view of this CERES-like instrument is thus shaped by the field stop instead of by the geometry of the telescope. This is in contrast with the ERBE scanning instrument, whose optical PSF is dominated by the telescope optics. This is an important characteristic for a scanning radiometer because diamond-shaped field stops can reduce both aliasing and blur ${ }^{25}$ when compared with circular apertures. We verify that the out-of-field radiation is of the order of only a few percent by integrating the PSF over the physical dimensions of the field stop. A more accurate estimate of the out-of-field radiation requires much greater resolution $\left(0.01^{\circ}\right.$ increments $)$ in the discretization of the field of view.

\section{Distribution of Energy on the Detector}

To couple the optical module to the electrothermal module, the distribution of radiation that arrives from the field on the active detector must be computed. The spatial distribution is discretized arbitrarily through a $16 \times 16$ grid. This provides sufficient spatial resolution without requiring excessive computational resources. For each of the 256 elements, the radiative power absorbed in the absorber layer, $P_{\text {elt }}$ bin , is computed as the product of the radiative energy incident upon the instrument aperture and the corresponding distribution factor:

$$
P_{\text {elt }}^{\text {bin }}=S^{\text {bin }} D_{\text {elt }}^{\text {bin }},
$$

where the subscript elt refers to the elements of the detector grid, the superscript bin refers to the angular bin defined by the cross-scan and scan angles, $S^{\text {bin }}$ is the power at the aperture associated with the point source for a given angular bin, and $D_{\text {elt }}^{\text {bin }}$ is the distribution factor defined in Eq. (17). It is important to note that $P_{\text {elt }}^{\text {bin }}$ is indeed the power absorbed by the detector element, as opposed to the incident power, because the absorption coefficient of the detector is a surface property that is taken into account in the ray-trace analysis of the optics module that defines $D_{\text {elt }}$ bin. The total power absorbed by the detector is

$$
P^{\text {bin }}=\sum_{\text {elt }} P_{\text {elt }}^{\text {bin }}
$$

Because the radiation incident upon the instrument within its field of view is defined as a radiance $\left(\mathrm{W} / \mathrm{m}^{2} \mathrm{sr}\right)$, it is convenient to compute an equivalent radiance corresponding to the total power absorbed by the detector. To do that we first compute a radiance equivalent to the power incident upon the aperture for each angular bin,

$$
L^{\text {bin }}=\frac{S^{\text {bin }}}{\Delta \Omega^{\text {bin }} A_{\text {ap }}}
$$

where $\Delta \Omega^{\text {bin }}$ is the solid angle defined in Eq. (16) and $A_{\text {ap }}$ is the surface area of the instrument aperture. The total equivalent radiance at the aperture is computed as a weighted average of the radiances in each angular bin, where the weighting factor is the distri- 

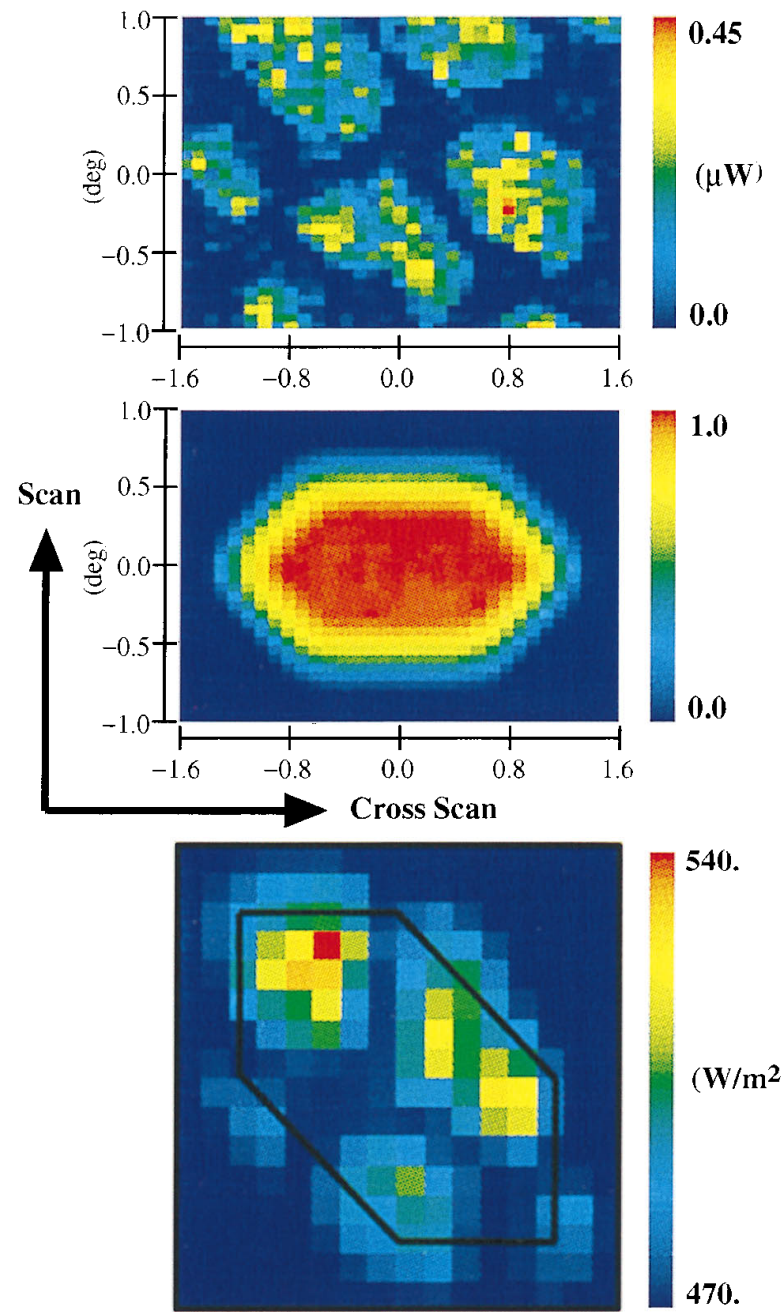

Fig. 8. Three stages in Earth scene image processing with the use of the optics module: (top) angular distribution of power at the aperture of the radiometer, (middle) PSF of the optics, (bottom) spatial distribution of radiant flux on the detector.

bution factor from the aperture to the detector in the given angular bin,

$$
L_{\text {eq }}=\frac{\sum_{\text {bin }} L^{\text {bin }} D^{\text {bin }}}{\sum_{\text {bin }} D^{\text {bin }}} .
$$

In Eq. (22), $D^{\text {bin }}$ is the total distribution factor from the aperture to the detector in a given angular bin, as defined in Eq. (18). Note that if the power incident upon the instrument aperture is uniform, the equivalent radiance in Eq. (22) will be equal to any of the bin radiances defined in Eq. (21).

Figure 8 shows three stages-input, transfer function, and output-in Earth scene image processing with the optics module. Figure 8(a) shows the angular distribution of power at the aperture of the radiometer produced from an Earth scene that represents solar radiation reflected from an ocean surface under $30 \%$ cloud cover. The cloud cover consists of large, low-altitude clouds, comparable to stratocumulus clouds. The data are obtained from a ray-trace model developed by Villeneuve. ${ }^{26}$ Each pixel in Fig. 8(a) corresponds to an angular bin. The value associated with each pixel is a power instead of a radiance because power is a quantity required by the electrothermal module. However, because of the direct correspondence between power and radiance defined in Eq. (21), this figure can be thought of as an image of the cloud scene. The equivalent radiance associated with this scene is approximately $125 \mathrm{~W} /$ $\mathrm{m}^{2} \mathrm{sr}$. Figure $8(\mathrm{~b})$ represents the PSF of the optics, which has already been depicted in Fig. 7. The data shown in Fig. 8(a) are used as input to the transfer function of Fig. 8(b) to produce the radiant flux absorbed on the detector surface. The spatial distribution of the absorbed flux discretized on a $16 \times 16$ grid is depicted in Fig. 8(c) along with the geometrical limits of the field stop. In addition to defining sharply the field of view of the instrument, the field stop also effectively limits the illuminated area of the detector to ensure all the radiation in the field of view can be detected. Note that the distribution of energy in the plane of the detector is an inverted image of the cloud scene because the detector is located behind the focal point of the instrument. This demonstrates the capability of the optical model to produce a twodimensional distribution of absorbed energy on the detector given any heterogeneous Earth scene.

\section{B. Predicted Dynamic Electrothermal Performance}

The dimensions of the various layers of the active and the compensating detectors and their thermophysical properties are given in Ref. 27, as are the values of the electrical components in Fig. 3. Nominal values for the CERES detector were used to obtain the results reported here.

\section{Temperature Distribution through the Detector}

The electrothermal module predicts the transient distribution of temperature throughout the entire detector unit, including both thermistor layers and their substrates. At each time step a vertical temperature profile through the center of the detector unit may be extracted from the complete temperature distribution. Figure 9(a) shows a temperature profile through the center of the active detector that corresponds to the detector at equilibrium while cold space is viewed. This temperature distribution is used as the initial condition for most simulations. Figure 9(b) shows the evolution with time of the vertical temperature profile through the center of the active detector after a $300-\mathrm{W} / \mathrm{m}^{2}$ sr step input. Note that only the part of the detector above the thermal impedance, i.e., the flat part of Fig. 9(a), is depicted in Fig. 9(b) to show temperature changes in the thermistor. Hence the bottom curve of Fig. 9(b) is a detail of Fig. 9(a).

Up to a time of $t=0$ the detector is at equilibrium while cold space is viewed. During this period, a portion of the Joulean heating resulting from bias current flowing through the thermistor is conducted to the left through a bonding layer and the absorber 


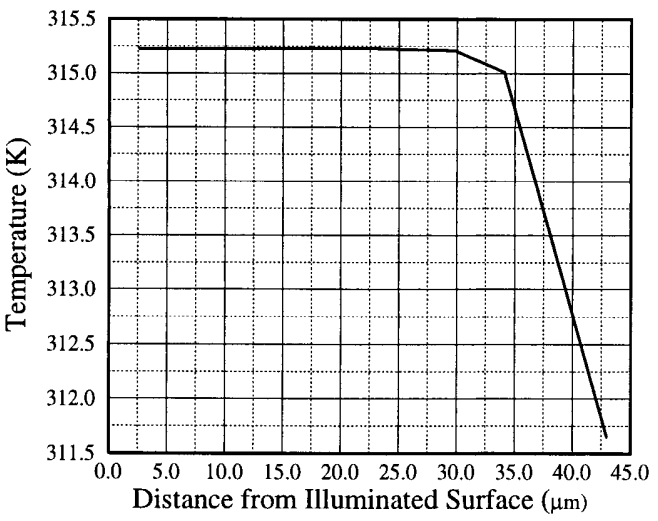

(a)

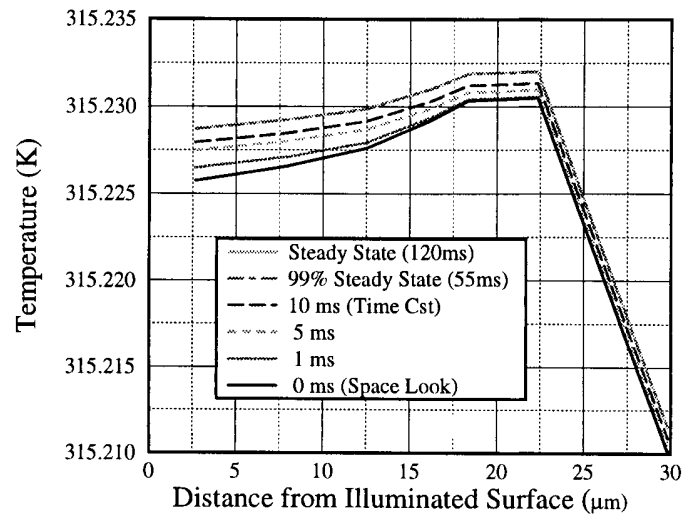

(b)

Fig. 9. Vertical temperature profile through the center of the detector unit: (a) the flake temperature at equilibrium while cold space is viewed, (b) evolution with time after a $300 \mathrm{~W} / \mathrm{m}^{2}$ sr step input.

layer and, subsequently, radiated into space, while the remainder of this self-heating is conducted to the right through another bonding layer and the thermal impedance layer and into the aluminum substrate. These two components of heat flux are manifested in Fig. 9 by downward-sloping line segments, sloping away from a relatively flat plateau near the center of the graph. The plateau represents the essentially isothermal thermistor layer. A constant irradiance-corresponding to a $300-\mathrm{W} / \mathrm{m}^{2} \mathrm{sr}$ radiance at the instrument aperture-is then applied to the detector at $t=0$, and the thermistor temperature (the flat plateau) begins to rise as less net heat is conducted to the left and radiated from the absorber. Note that the temperature axis covers a range of only a few hundredths of a degree and the thermistor temperature changes only approximately $3 \mathrm{mK}$.

Figure 10(a) shows the evolution with time of the spatially averaged thermistor temperatures in both the active and the compensator detectors, corresponding to the conditions of Fig. 9. Up to a time of $t=0$, both detectors have similar temperatures. Beyond this time the temperature in the active detector

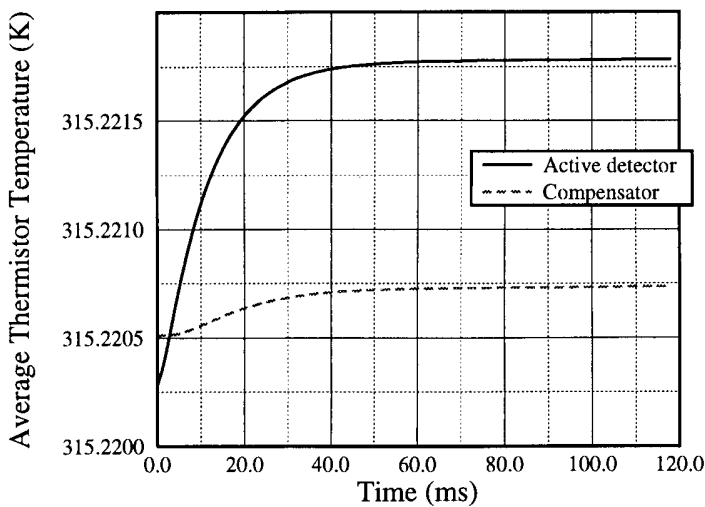

(a)

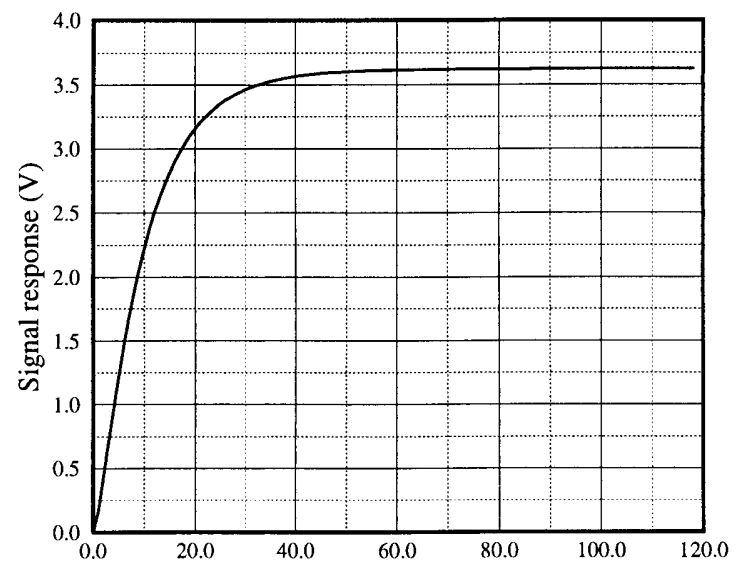

(b)

Fig. 10. Evolution with time after a $300 \mathrm{~W} / \mathrm{m}^{2}$ sr step input of (a) the spatially averaged thermistor temperatures in both the active and the compensator detector flakes, (b) the signal response of the instrument.

increases owing to the radiative input. At this point, changes in the thermal environment can also induce temperature changes in the active detector. The temperature of the compensator detector also changes under the influence of the changing electrical conditions in the external circuit (self-heating). In a previous study, ${ }^{13}$ Haeffelin showed the time constant of the detector is $8.5 \mathrm{~ms}$ when only a radiative step input is considered. Here the time constants of the active and the compensating detectors are, respectively, $12 \mathrm{~ms}$ and $22 \mathrm{~ms}$, assuming a first-order time response. Clearly the temperature changes in Fig. 10(a) do not correspond to first-order time responses because the energy inputs are not step inputs. For the active detector it is a combination of a radiative step input with a slowly drifting internal source, and for the compensating flake it is simply a changing internal source.

Figure 10(b) shows the evolution with time of the signal response of the instrument to a $300-\mathrm{W} / \mathrm{m}^{2} \mathrm{sr}$ step input. The time response can be fitted with a 
first-order function and the resulting time constant becomes $10 \mathrm{~ms}$, which is in the same range as the experimentally determined instrument time constant of the CERES detectors. Hence the model shows that thermal changes in the environment, whether caused by self-heating or other sources, that would affect the response of the active detector are effectively compensated by a matching compensating detector. This is gratifying because nominal dimensions and properties and component values furnished by the manufacturer were used in the model; but otherwise the model result is completely independent of the experimental result.

\section{Study of Equivalence}

The concept of equivalence, or nonequivalence, refers to the effect that spatial distribution of a given radiative power input to the instrument might have on the instrument signal response. The equivalence of a scanning radiometer is an important concept because Earth scenes and calibration scenes of equivalent power are likely to produce images in the detector plane with different spatial distributions. In a previous study by Haeffelin, ${ }^{13}$ the author showed that the hypothesis of equivalence for the ERBE scanning radiometer was a good assumption and that the instrument probably is not sensitive to the type of differences in Earth radiative fields that would typically be encountered in practice. However, the study did not include the optics module, and the equivalence tests were based on absorbed radiative power on the detector instead of equivalent radiance at the instrument aperture.

a. Response of Detector to Spot Illuminations: A numerical experiment was performed to simulate a laser point heating experiment performed by the constructor. A $10-\mu \mathrm{W}$ spot heating is applied sequentially to each of the 256 surface elements into which the absorber layer has been divided. The spot size is approximately $0.1 \mathrm{~mm} \times 0.1 \mathrm{~mm}$. For each illumination the detector is allowed to evolve from initial conditions, corresponding to a space look, to steadystate conditions.

Figure 11 shows the predicted normalized steadystate detector response, corresponding to the laser point heating numerical experiment. Figure 11(a) depicts a one-dimensional representation of the distribution; Fig. 11(b) is a two-dimensional representation. The predicted instrument sensitivity to spot illumination is quite uniform over a large region in the center of the detector. The response to spot heating falls off only near the edges to about $70 \%$ of the center value. The average normalized response is approximately 0.94 . Figure $11(\mathrm{~b})$ also shows the geometrical shape of the field stop. Note that the spatial distribution of absorbed energy depicted in Fig. 8(c) falls within a region in which the normalized responsivity of the detector is greater than 0.9 .

b. Response of Detector to Nonuniform Earth Scenes: The combined optical and electrothermal modules are used to compute the steady-state signal response of the instrument to the nonuniform Earth

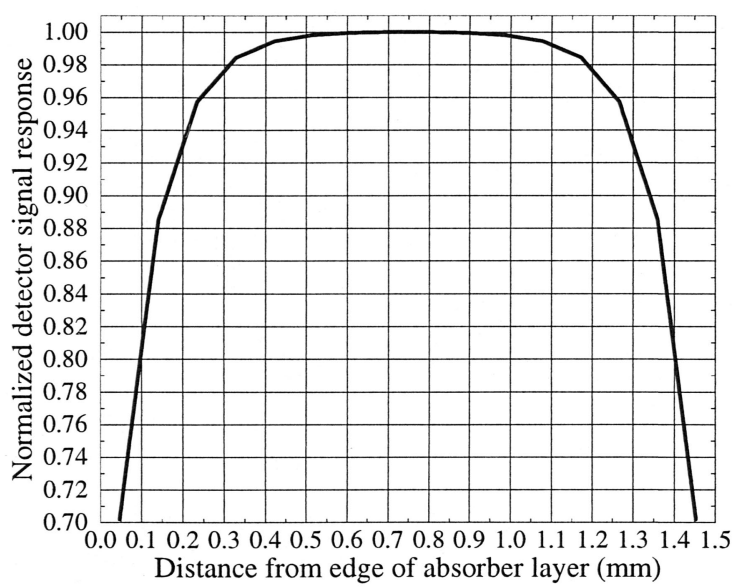

(a)

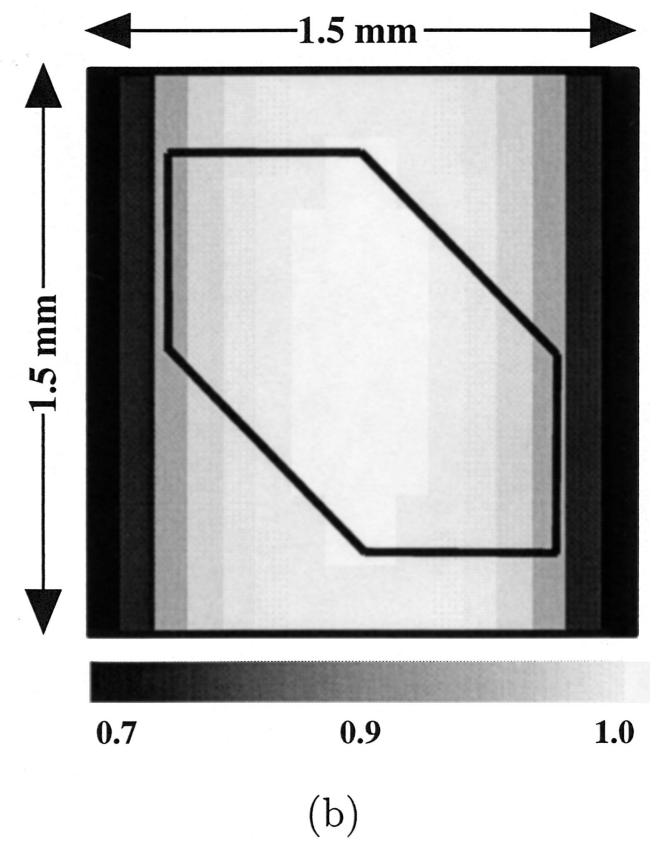

Fig. 11. (a) One-dimensional representation, (b) two-dimensional representation of the distribution of the normalized steady-state signal response to $10-\mu \mathrm{W}$ spot illuminations.

scene shown in Fig. 8(a). This scene represents large decks of low stratiform clouds over an ocean surface. The input equivalent radiance of this nonuniform scene is $126.68 \mathrm{~W} / \mathrm{m}^{2} \mathrm{sr}$. Given the radiative input of Fig. 8(a), the combined optical and electrothermal modules compute a steady-state output of $1.505 \mathrm{~V}$. We can convert the output signal into an output equivalent radiance by applying a voltage-to-radiance calibration based on viewing a diffuse calibration source. ${ }^{23}$ This value is $126.29 \mathrm{~W} /$ $\mathrm{m}^{2} \mathrm{sr}$, which represents a $0.3 \%$ difference from the input radiance. This predicted difference can be interpreted in terms of equivalence between a uniform calibration scene and a typical nonuniform Earth 
scene. The instrument reveals a high level of equivalence.

\section{Instrument Point-Spread Function}

An important part of the radiometric data analysis is to take into account and compensate for the time response of the detector and the electronic circuit. Compensation involves shifting the pixel location by a given amount with respect to the instantaneous location of the optical axis. The shift of the pixel is defined by the scanning instrument PSF, which can be computed as the product of the scan rate, the detector time response, and the optical PSF. ${ }^{28,29}$ In Manalo et al., ${ }^{28}$ the optical PSF was taken to be governed by the field stop, and the detector response was assumed to be first order.

With the CERES-like end-to-end model, we can compute an instrument-like PSF by scanning across a uniform line source of radiation, which is a distribution of point sources along a line normal to the scan direction, and recording the transient response of the model. In the numerical experiment, each point source produces an output signal that is independent of that of the other point sources. Note that the electronic circuit that has been modeled is as shown in Fig. 3 and hence does not include the four-pole Bessel filter used in the actual CERES instrument. Therefore the results presented here represent only the PSF through the preamplifier electronics. Significant modulation of the signal can occur, depending on the filter characteristics.

Figure 12 shows an instrument-like PSF as a function of the position in the scan and cross-scan directions. Figure 12(a) shows detailed distributions with scan angles for several values of the cross-scan angle. Figure 12(b) shows a two-dimensional representation of the distribution in which the shift with respect to the optical axis $-0.0^{\circ}$ in both scan and cross-scan anglesappears clearly. Because of the thermal and electrical inertia of the sensing element and signalconditioning circuit, the tail of the distribution goes out to approximately $4.0^{\circ}$ beyond the optical axis of the instrument, which corresponds to approximately 63 $\mathrm{ms}$ because the scan rate is $63.5 \mathrm{deg} / \mathrm{s}$.

During the processing the radiometric data, the shift of the pixel is taken to be the centroid of the PSF with respect to the origin. ${ }^{28}$ The centroid in the scan direction, $\zeta_{\text {cen }}$ is defined as the weighted average of the scan angles in Fig. 12(b), where the weighting function is the PSF, i.e.,

$$
\zeta_{\text {cen }}=\frac{\sum_{i} \zeta(i) \sum_{j} \operatorname{PSF}(i, j)}{\sum_{i} \sum_{j} \operatorname{PSF}(i, j)} .
$$

In Eq. (23), $i$ and $j$ are the indices in the scan and cross-scan directions, respectively, $\zeta(\mathrm{i})$ is the angular position in the scan direction, and $\operatorname{PSF}(i, j)$ is the $i, j$ value of the PSF.

In the current configuration of the optics and the electrothermal models, the centroid is found to be
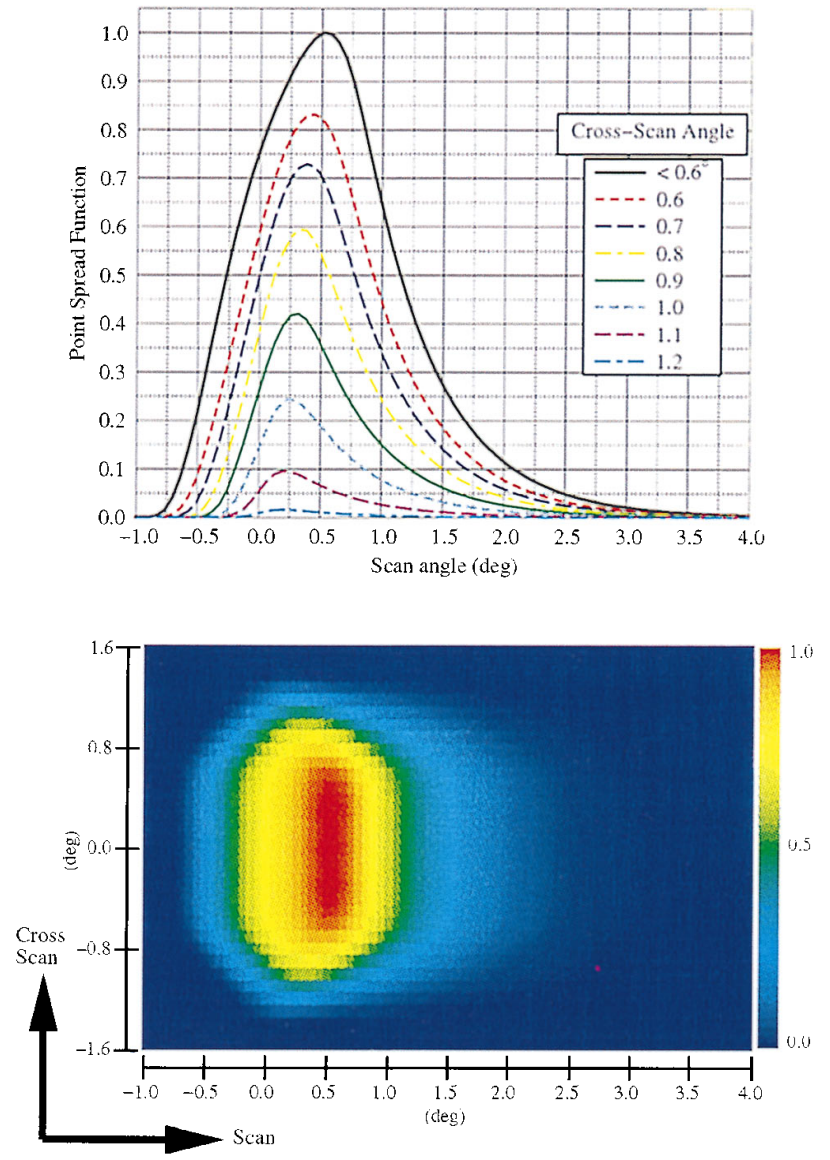

Fig. 12. Instrument PSF: (top) one-dimensional representation and (bottom) two-dimensional representation.

approximately $0.63^{\circ}$, or $10 \mathrm{~ms}$, behind the origin. This important result is needed for the data processing associated with simulation of Earth scans. Note that a centroid has been computed only in the scan direction because the scan rate produces a scan velocity that is much greater than the velocity of the satellite. The linear velocity of the satellite is approximately $7 \mathrm{~km} / \mathrm{s}$ or $7 \mathrm{~m} / \mathrm{ms}$, which is equivalent to an angular rate of $5 \times 10^{-4} \mathrm{deg} / \mathrm{ms}$ in the cross-scan direction. During the time it takes to integrate the signal of the field of view, $100 \mathrm{~ms}$, for example, the cross-scan angle will have moved $0.05^{\circ}$, which is twice the resolution of a cross-scan angular bin. Therefore this motion can be neglected in the process of converting the instrument output signal into radiance at the aperture.

\section{Conclusions}

A high-level end-to-end numerical model of the scanning thermistor bolometer radiometers planned for use in CERES is presented, and two modules are described in some detail. Results from the opticalthermal radiative module establish that the instrument's optical system provides relatively uniform weighting over most of its footprint on the Earth while it uniformly distributes gathered radiation on the active detector. Very little out-of-field radiation 
reaches the active detector. The thermistor bolometer dynamic electrothermal module accurately predicts the instrument frequency response characteristics while it lends considerable insight into its operation. It also provides convincing data, indicating that uncertainties associated with nonequivalence are negligible. The two modules are interconnected so that the optical-thermal radiative module can scan a given scene to present a time series of inputs to the thermistor bolometer dynamic electrothermal module. This capability is used to define the instrument PSF taking into account both the instrument optics and the thermal and electronic time responses of the detector unit. The potential for using this combination of modules to evaluate ground and on-board calibration procedures and even to validate data-reduction strategies is obvious.

The authors acknowledge the Radiation Sciences Branch of the Atmospheric Sciences Division at NASA's Langley Research Center for its support of this study under grant NAG-1-1456.

\section{References}

1. Inadvertent Climate Modification, Final Report of the Study of Man's Impact (MIT, Cambridge, Mass., 1971).

2. B. R. Barkstrom and G. L. Smith, "The Earth radiation budget experiment: science and implementation,” Rev. Geophys. 24, 379-390 (1986).

3. V. Ramanathan, R. D. Cess, E. F. Harrison, P. Minnis, B. R. Barkstrom, E. Ahmad, and D. Hartmann, "Cloud radiative forcing and climate: results from the Earth radiation budget experiment," Science 243, 57-63 (1989).

4. B. R. Barkstrom and J. B. Hall Jr., "The Earth radiation budget experiment (ERBE): an overview," J. Energy 6, 141-146 (1982).

5. B. R. Barkstrom, "The Earth radiation budget experiment (ERBE)," Bull. Amer. Meteorol. Soc. 65, 1170-1185 (1984).

6. B. A. Wielicki and B. R. Barkstrom, "Clouds and the Earth's radiant energy system (CERES): an Earth observing system experiment," in Proceedings of the Second Symposium on Global Change Studies, E. J. Barron, J. R. Mahoney, C. B. Baker, R. J. Gurney, and J. T. Merrill, eds. (American Meteorological Society, Boston, Mass., 1991), pp. 11-16.

7. S. L. Carman, J. E. Cooper, J. Miller, E. F. Harrison, and B. R. Barkstrom, "Clouds and the Earth's radiant energy system (CERES),” Adv. Astrophys. Sci. 76, 693-706 (1992).

8. N. E. Tira, "Dynamic simulation of solar calibration of the total Earth-viewing channel of the Earth Radiation Budget Experiment (ERBE)," Master's thesis (Virginia Polytechnic Institute and State University, Blacksburg, Va., 1987).

9. N. E. Tira, "A study of the thermal and optical characteristics of radiometric channels for Earth radiation budget applications," Ph.D. dissertation (Virginia Polytechnic Institute and State University, Blacksburg, Va., 1991).

10. R. P. Bongiovi, "A parametric study of the radiative and optical characteristics of a scanning radiometer for Earth radiation budget applications using the Monte-Carlo method," Master's thesis (Virginia Polytechnic Institute and State University, Blacksburg, Va., 1993).

11. M. Walkup, "Creation of a Monte-Carlo optical work bench for radiometric imaging," Master's thesis (Virginia Polytechnic Institute and State University, Blacksburg, Va., 1996).

12. J. L. Meekins, "Optical analysis of the ERBE scanning thermistor bolometer using the Monte-Carlo method," Master's thesis (Virginia Polytechnic Institute and State University, Blacksburg, Va., 1990).

13. M. P. A. Haeffelin, "A numerical study of equivalence in scanning thermistor bolometer radiometers for Earth radiation budget applications," Master's thesis (Virginia Polytechnic Institute and State University, Blacksburg, Va., 1993).

14. K. J. Priestley, J. R. Mahan, and R. B. Lee III, "Use of highlevel numerical radiometric channel models for Earth radiation budget signal processing," presented at the Sixth Annual Inverse Problems in Engineering Seminar, University of Cincinnati, Ohio, 1994.

15. J. R. Howell, "Application of Monte Carlo to heat transfer problems," in Advances in Heat Transfer (Academic, New York, 1968), Vol. 5, pp. 1-54.

16. T. S. Chou, "A Monte Carlo approach to optical analysis," Opt. Eng. 13, 299-302 (1974).

17. J. R. Mahan and L. D. Eskin, "Application of the Monte Carlo technique to transient thermal modeling of cavity radiometers having diffuse-specular surfaces," in Proceedings of the Fourth Conference on Atmospheric Radiation, C. L. Mater, ed. (American Meteorological Society, Boston, Mass., 1981), pp. 181-186.

18. J. R. Mahan and L. D. Eskin, "The radiation distribution factor its calculation using Monte Carlo and an example of its application," in Proceedings of the First UK National Heat Transfer Conference, H. C. Simpson, ed., Report No. 19 (European Federation of Chemical Engineering Publication Series, Leeds, England, 1984), pp. 1001-1012.

19. J. R. Mahan, F. Kowsary, and L. D. Eskin, "Apparent emissivities of conical and cylindrical cavities: comparison of Monte Carlo and exact analytical results," in Radiation, Phase Change Heat Transfer, and Thermal Systems, Y. Jaluria, V. P. Carey, W. A. Fiveland, and W. Yuen, eds. (American Society of Mechanical Engineers (ASME), New York, 1987), pp. 1-8.

20. N. E. Tira, J. R. Mahan, and R. B. Lee III, "Dynamic electrothermal model for the ERBE nonscanning radiometer with application to solar observation and thermal noise," Opt. Eng. 29, 351-358 (1990).

21. N. E. Tira, J. R. Mahan, R. B. Lee III, and R. J. Keynton, "Linear-array apertures for in-flight dynamic solar calibration of radiometric channels for Earth radiation-budget applications," Appl. Opt. 33, 5617-5627 (1994).

22. S. V. Patankar, Numerical Heat Transfer and Fluid Flow (Hemisphere, New York, 1980).

23. M. P. A. Haeffelin, "A study of Earth radiation budget radiometric channel performance and data interpretation protocols," Ph.D. thesis (Virginia Polytechnic Institute and State University, Blacksburg, Va., 1996).

24. R. Siegel and J. R. Howell, Thermal Radiation Heat Transfer (Hemisphere, Washington, D.C., 1992).

25. S. J. Katzberg, F. O. Huck, and S. D. Wall, "Photosensor aperture shaping to reduce aliasing in optical-mechanical linescan imaging systems," Appl. Opt. 12, 1054-1060 (1973).

26. P. V. Villeneuve, "A numerical study of the sensitivity of cloudy-scene bidirectional reflectivity distribution functions to variations in cloud parameters," Ph.D. thesis (Virginia Polytechnic Institute and State University, Blacksburg, Va., 1996).

27. CERES Drawing Package: Encoder and Sensor Drawings (BEI, Servo, Adcole), TRW Applied Technology Division, Space and Electronics Group, One Space Park, Redondo Beach, Calif. 90278, 1993.

28. N. Manalo, G. L. Smith, and B. R. Barkstrom, "Transfer function considerations for the CERES scanning radiometer," in Proceedings of the Tenth Conference on Optoelectronics and Microwave, H. N. Nasr, ed. (International Society for Optical Engineering, Bellingham, Wash., 1991), pp. 106-116.

29. G. L. Smith, "Effects of time response on the point-spread function of a scanning radiometer," Appl. Opt. 33, 7031-7037 (1994). 